\title{
Primes and Targets in Rapid Chases: Tracing Sequential Waves of Motor Activation
}

\author{
Thomas Schmidt \\ University of Giessen
}

\author{
Silja Niehaus and Annabel Nagel \\ University of Göttingen
}

\begin{abstract}
Single-cell recordings have indicated that visual stimuli elicit rapid waves of neuronal activation that propagate so fast that they might be free of intracortical feedback. Here, the time course of feedforward activation was traced by measuring pointing responses to color targets preceded by color primes initiating either the same or opposite response. The early time course of priming effects was strictly time locked to prime onset and depended only on properties of the primes, but was independent of the onset times of the actual targets as well as the perceptual effects of targets on primes. Results indicated that nonoverlapping feedforward signals by primes and targets traverse the visuomotor system in a rapid chase, controlling associated motor responses in strict sequence.
\end{abstract}

Keywords: response priming, masked priming, feedforward sweep, pointing movements, autopilot
In a meta-analysis of 48 studies investigating the response times of various cortical areas to a sudden visual stimulus, Lamme and Roelfsema (2000) showed that the stimulus creates a wave of activation traveling from posterior to anterior areas, reaching most cortical areas within about $150 \mathrm{~ms}$, including primary motor and premotor cortices. Lamme and Roelfsema estimated that this leaves cells with only about $10 \mathrm{~ms}$ of time to pass their own activation on to later areas, which is about the duration of a typical interspike interval. Because most cells have to pass on their activation with the next spike fired, there is little or no time to integrate feedback from other cells. On the basis of this finding, Lamme and Roelfsema suggested that the first wave of visual activation travels through the system as a fast feedforward sweep that is essentially free of intracortical feedback information. Indeed, neural network simulations of rapid stimulus classification tasks suggest that most of the stimulus-relevant information could be extracted from the temporal distribution of the very first spikes in the feedforward wavefront (Rousselet, Fabre-Thorpe, \& Thorpe, 2002; VanRullen \& Thorpe, 2002), showing that purely feedforward mechanisms are capable of fast and efficient processing. ${ }^{1}$

If the notion of a feedforward sweep is applied strictly, each cell first reached by the feedforward wavefront can respond to it only on the basis of its preestablished input-output properties. The feedforward hypothesis in this strong form is controversial, because feedback mechanisms in early visual areas can be very rapid (Bullier, 2001; Girard, Hupé, \& Bullier, 2001), and there are many possibilities for signals processed in parallel visual streams to

Thomas Schmidt, Department of Psychology, University of Giessen, Giessen, Germany; Silja Niehaus and Annabel Nagel, Department of Psychology, University of Göttingen, Göttingen, Germany.

This work was supported by the German Research Foundation Grant Schm1671/1 to Thomas Schmidt.

Correspondence concerning this article should be addressed to Thomas Schmidt, Department of Psychology, University of Giessen, Otto-BehaghelStr. 10F, D-35390, Giessen, Germany. E-mail: thomas.schmidt@psychol.unigiessen.de cross or overtake each other well before the first overt signs of motor activation might occur (Merigan \& Maunsell, 1993). Moreover, Lamme and Roelfsema (2000) based their arguments on the minimum cortical activation times reported in their sample studies; average activation times are actually $30-70 \mathrm{~ms}$ larger and show considerable variation across the studies in the sample. This makes the feedforward hypothesis vulnerable for attack.

However, it would be premature to dismiss the notion of an initial feedforward phase in human brain activation just because it may not be entirely free of intracortical feedback. Our strategy in this article is to look at the input-output properties of the visuomotor system as a whole to evaluate some behavioral predictions of the feedforward sweep hypothesis. To do this, we introduce the concept of a rapid chase. This concept applies to visuomotor tasks in which the system responds to successive visual stimuli that simultaneously run for control of a single speeded motor response, for example, when a pointing response is initiated by one stimulus and then altered mid-flight by an immediately following stimulus (e.g., Brenner \& Smeets, 2004; Schmidt, 2002). By our definition, two successive visuomotor signals are said to be engaged in a rapid chase if (a) the response is initiated by the first stimulus, (b) the response is influenced by the second stimulus before it is completed, and (c) the response to the first stimulus is initially independent of the second stimulus (rapid chase criteria). In other words, if successive signals are in a rapid chase, they will take successive control over the same motor response, but there will be

\footnotetext{
${ }^{1}$ This usage of the term feedforward is different from that in control theory, which distinguishes between feedforward and feedback control. In the context of motor control of a pointing movement, feedforward (open loop) control means that the movement, once started, unfolds without incorporating external feedback. In contrast, if feedback (e.g., about the current distance to the target position) is incorporated during the movement, the movement is part of a closed loop. In the context of neuronal signal flow, however, feedforward means that a cell passes activation on to another cell before integrating any feedback from other cells about its own signal (VanRullen \& Koch, 2003). It is this usage we follow here.
} 
a time period when the response is exclusively controlled by the first stimulus. The notion of a rapid chase is milder than the feedforward sweep hypothesis: Whereas the feedforward sweep entails the possibility of rapid chases, the rapid chase account allows for recurrent activity as long as sequential signals still lead to strictly sequential motor output. In this article, we present behavioral evidence for the existence of rapid chases obeying the above criteria, yielding independent behavioral evidence consistent with Lamme and Roelfsema's (2000) claim of a fast feedforward sweep.

Identifying tasks in which processing is exclusively feedforward and nonrecurrent is theoretically interesting because several authors have proposed that conscious perception is possible only with recurrent processing (e.g., DiLollo, Enns, \& Rensink, 2000; Lamme, 2000). Evidence for this view comes from studies indicating that visual awareness of a stimulus is suppressed if feedback loops from extrastriate visual areas through primary visual cortex are disrupted at critical points in time, for example, by a visual backward mask (Lamme, Zipser, \& Spekreijse, 2002; Macknik \& Haglund, 1999; Macknik \& Livingstone, 1998) or by transcranial magnetic stimulation (Pascual-Leone \& Walsh, 2001; Ro, Breitmeyer, Burton, Singhal, \& Lane, 2003). If some tasks were known to be independent of recurrent processing, such feedforward tasks might be used to study the properties of unconscious processing.

We used a response priming paradigm (Vorberg, Mattler, Heinecke, Schmidt, \& Schwarzbach, 2003; see also Ansorge \& Neumann, 2005; Klotz \& Neumann, 1999; Neumann \& Klotz, 1994) to demonstrate rapid chases because there is strong evidence that this paradigm involves rapid and successive response activation that is independent of visual awareness. In a typical response priming experiment, Vorberg et al. (2003) had participants perform keypress responses to the direction of large arrow targets that were preceded by small arrow primes, the pointing direction of which could be either the same as the target's (consistent primes) or opposite to the target's (inconsistent primes). The large arrows served a dual purpose (Neumann \& Klotz, 1994): They were the targets for the keypress response and also served to reduce the visibility of the primes by metacontrast, a form of visual backward masking (Breitmeyer, 1984; Francis, 1997). Responses were speeded by consistent primes and slowed by inconsistent primes, and the magnitude of this priming effect increased linearly with the stimulus onset asynchrony (SOA) of prime and mask. Strikingly, the time course of priming was independent of the visibility of the prime: The increase in priming with SOA was the same regardless of whether the prime could be identified perfectly or not at all, and regardless of whether prime identification performance increased or decreased with SOA. It has been shown that primes directly trigger the specific motor responses assigned to them, leading to a shift in lateralized readiness potentials (Eimer \& Schlaghecken, 2003; Leuthold \& Kopp, 1998; Vath \& Schmidt, 2006; Verleger, Jaskowski, Aydemir, van der Lubbe, \& Groen, 2004) and lateralized metabolic activity in motor areas (Dehaene et al., 1998).

The time course of visuomotor control has been further examined in primed pointing responses by Schmidt (2002). In that study, participants were presented with one red and one green prime flashed simultaneously in diagonally opposite quadrants of the display, followed by one red and one green metacontrast mask (annuli that closely fitted around the primes) at the same locations as the primes. Either primes and masks at corresponding positions could have the same colors (consistent primes), or prime colors could be switched compared with mask colors (inconsistent primes). Participants had to point as quickly as possible from the fixation point toward the mask with the appointed color (mask identification [ID] task, designed to measure response priming effects) or try to point without time pressure to the position where they believed the prime of that color had occurred (prime ID task, designed to assess visual awareness of the primes). Results strongly suggested that responses were controlled consecutively by prime and mask signals even when the primes were completely masked. Pointing responses started at a fixed time following prime onset and initially went into the direction specified by the primes, even though the mask signals were the actual targets of the response. When primes and masks were consistent, this initial direction was correct, and the finger simply traveled toward the correct mask until the response was completed. When primes and masks were inconsistent, however, the finger initially traveled into the quadrant occupied by the misleading prime. This detour into the wrong quadrant lasted for a time directly depending on primemask SOA; then the finger stopped and finally proceeded in the correct direction. These data suggest that pointing movements are under continuous control of the color stimuli responded to: Prime signals reach motor areas of the brain in advance of the mask signals, governing the initial phase of the pointing response, whereas mask signals are able to take control mid-flight, with a delay depending on the prime-mask SOA.

We wondered whether this continuous flow of visuomotor information might conform to our definition of a rapid chase, such that the initial motor activation triggered by the primes would be independent of the mask stimuli. To examine this, we used Schmidt's (2002) pointing paradigm, varying the overall feedforward dynamics of prime and mask signals by presenting all stimuli with either low or high color contrast and leaving their subjective luminance constant. We further varied the perceptual effects of the masks by using either efficient metacontrast masks with contours adjacent to those of the primes (strong masks), or pseudomasks that did not share contours with the primes (weak masks; see Figure 1). If motor responses were controlled sequentially by prime and mask signals engaged in a rapid chase, their early time courses should exclusively depend on properties of the primes, but be invariant with respect to any properties of the masks. Moreover, if the rapid chase was based on feedforward processing whereas visual awareness of the primes required recurrent processing, early motor activation by the primes should be independent of the masks' perceptual effects on the primes. We performed two experiments that were identical except for the prime-mask SOA range covered: 33-100 ms in Experiment 1, and $17 \mathrm{~ms}$ in Experiment 2 .

\section{Method}

\section{Participants}

Six students from the University of Göttingen (Experiment 1, ages 20-25 years, two were left-handed, all were female; Experiment 2, ages 19-23 years, two were left-handed, one was male, and five were female) volunteered for course credits or for a payment of $€ 48$ (U.S. \$48). Their vision was normal or corrected to normal. All of them gave informed consent. Data from one participant in Experiment 2 had to be dismissed because she did not meet a preset accuracy criterion in mask ID. 


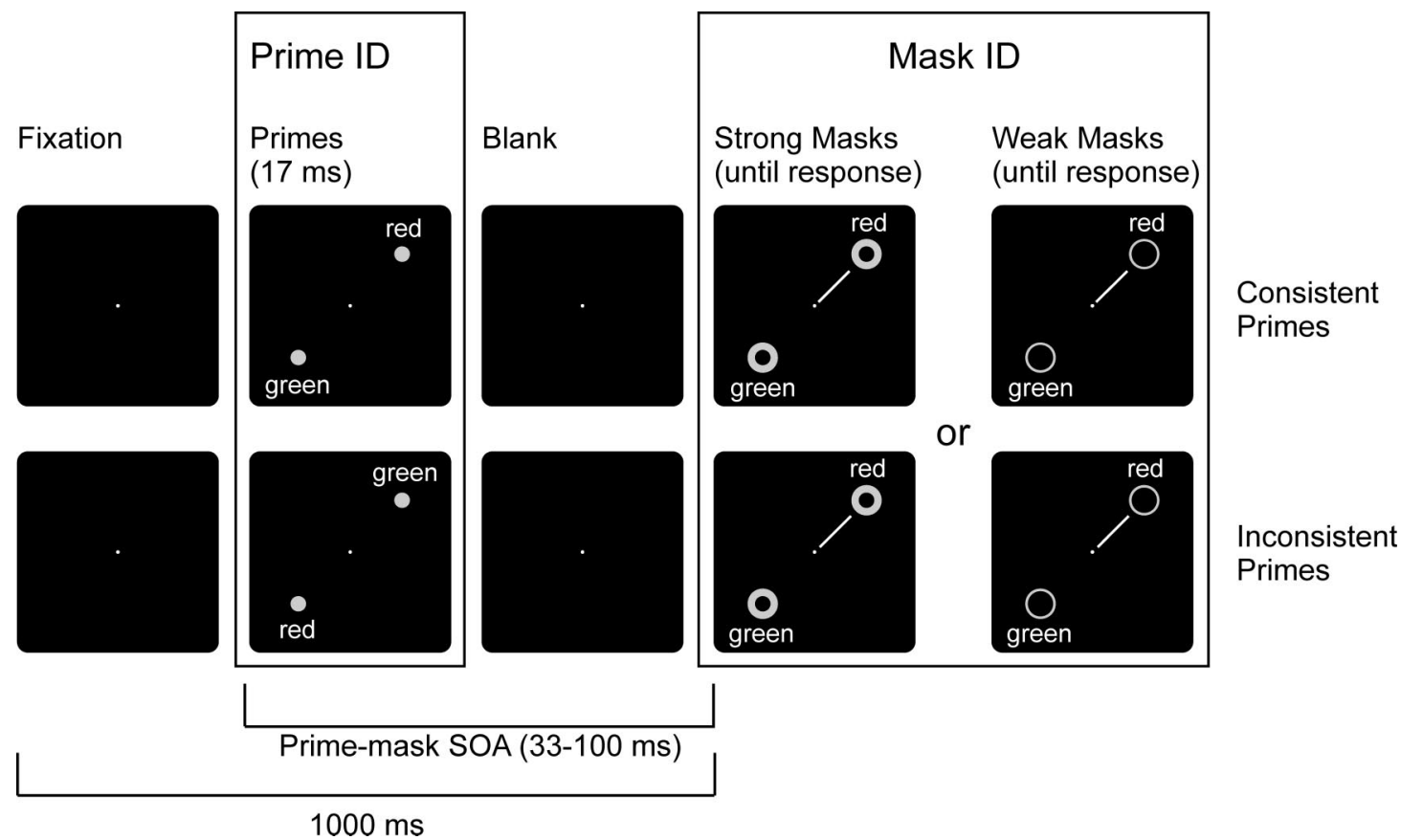

Figure 1. Stimuli and procedure. Two example trials are shown.

\section{Apparatus}

The experiment was controlled by a $300-\mathrm{Mhz}$ PC driving a 14-in. VGA color monitor $(640 \times 480$ pixels $)$ in synchrony with the monitor retrace rate of $60 \mathrm{~Hz}$. The monitor image was projected onto a workspace via a set of two mirrors, such that participants had the impression that the stimuli appeared directly on the workspace where they could interact with them. The workspace was tilted toward the participant by $44^{\circ}$ out of the transversal plane at $70 \mathrm{~cm}$ viewing distance and was illuminated such that participants could view their finger superimposed on the stimuli. Pointing responses were recorded by a Polhemus (Colchester, VT) FASTRAK magnetic tracking device at a sampling frequency of $120 \mathrm{~Hz}$. The sensor was a small cubicle of about $1 \mathrm{~cm}^{3}$ taped to the tip of the index finger of the preferred hand.

\section{Stimuli}

Primes were small disks $\left(7.6 \mathrm{~mm}\right.$ diameter; $1 \mathrm{~mm} \approx 0.08^{\circ}$ of visual angle) presented against a dark background $\left(0.07 \mathrm{~cd} / \mathrm{m}^{2}\right), 51.2 \mathrm{~mm}$ from a $83.00 \mathrm{~cd} / \mathrm{m}^{2}$ fixation point $(1.4 \mathrm{~mm}$ diamter). Masks were annuli with an outer diameter of $14.9 \mathrm{~mm}$ and an inner diameter of either $13.1 \mathrm{~mm}$ (weak masks) or of the size of the primes (strong masks). Isoluminant red and green colors were determined by heterochromatic flicker photometry for each participant. Low-contrast reds had $21 \% \pm 2 \%, 9 \%$, and $10 \%$ of the maximum luminances in the monitor's red, green, and blue channels, respectively (between-subjects means and standard deviations are given for those values adjusted by the participants). Corresponding values for highcontrast reds, low-contrast greens, and high-contrast greens were (a) $43 \%$ $\pm 17 \%, 3 \%, 5 \%$; (b) $13 \%, 12 \% \pm 0 \%, 10 \%$; and (c) $6 \%, 15 \% \pm 1 \%, 5 \%$, respectively. All colors were matched to the same standard gray ( 4.53 $\left.\mathrm{cd} / \mathrm{m}^{2}\right)$.

\section{Procedure}

In each trial, participants had to place their finger on the fixation point at display center and to press the space bar to initiate a trial (Figure 1).
They were then presented with one red and one green prime flashed simultaneously for $17 \mathrm{~ms}$ in diagonally opposite quadrants of the display. The quadrant where the red prime was presented was determined randomly in each trial. After a prime-mask SOA of variable duration (Experiment 1, 33, 67, or $100 \mathrm{~ms}$; Experiment 2, $17 \mathrm{~ms}$ ), one red and one green mask were presented at the same locations as the primes such that their colors were either consistent or inconsistent (switched) with respect to the prime colors. They remained on screen until participants had either finished a speeded pointing response towards the mask with stipulated color (mask ID task) or had pointed without time pressure to the position where they believed the prime of that color had appeared (prime ID task). Arrival time was defined as the time when the sensor first entered a sphere of $20 \mathrm{~mm}$ radius around any of the four target positions for at least $50 \mathrm{~ms}$.

Participants took part in eight experimental sessions. Half of each session was prime ID, the other half mask ID, with the order of tasks counterbalanced across participants and sessions. Each half of the session consisted of one warm-up block followed by nine experimental blocks of 32 trials, and stimulus conditions occurred equiprobably and pseudorandomly in a completely crossed repeated-measures design. The first session was preceded by the flicker photometry task and by a short slow-motion demonstration program with clearly visible primes where both prime and mask ID could be practiced. Each participant responded to only one color (either red or green, counterbalanced across participants) throughout all tasks and sessions. Participants were debriefed after the final session and received an explanation of the experiment.

\section{Data Treatment and Statistical Methods}

Four sessions of Experiment 1 were lost because of equipment malfunction. Practice blocks were not analyzed. Trials were excluded if the participant had hit one of the empty, off-diagonal target locations, or if arrival times were shorter than $100 \mathrm{~ms}$ or longer than either $999 \mathrm{~ms}$ (mask ID) or 4,999 ms (prime ID). The procedure eliminated $4.08 \%$ of trials in Experiment 1, mainly from one participant in prime ID, and $0.97 \%$ of trials in Experiment 2. Rates of correct identification responses in prime ID were 
arcsine-transformed to fit analysis of variance (ANOVA) requirements and backed up with signal-detection statistics (not reported here). All ANOVAs are reported with Greenhouse-Geisser-corrected $p$ values.

\section{Results and Discussion}

\section{Experiment 1}

Arrival times. Primes had large effects on speeded responses to the masks. Arrival times at the correct target location were considerably shorter in consistent than in inconsistent trials, and arrival times in consistent trials strongly decreased with primemask SOA. This decrease was much steeper for high-contrast than for low-contrast stimuli. Overall, arrival times were shorter for high-contrast than for low-contrast stimuli, and also slightly shorter for strong than for weak masks. A similar pattern of effects was observed in the error rates: Erroneous responses in direction of the incorrect target ran to completion in inconsistent trials only, that is, when that quadrant was occupied by the misleading prime. Error rates increased with SOA, the increase being steeper for high-contrast stimuli and weak masks.

Response priming effects (defined as arrival times in inconsistent minus consistent trials) were analyzed with a repeatedmeasures ANOVA with factors of SOA, contrast, and mask type (see Figure 2A, SOAs of 33-100 ms). Pooled over all conditions, there was strong evidence for response priming as indicated by the large intercept effect, $F(1,5)=174.86, p<.001$. Priming strongly increased with SOA, $F(2,10)=94.79, p<.001$; was stronger for high-contrast than for low-contrast stimuli, $F(1,5)=15.66, p=$ .011 ; and was stronger for weak than for strong masks, $F(1,5)=$ $9.86, p=.026$. Bonferroni-corrected planned contrasts revealed that the effect of mask type was significant only for high-contrast but not for low-contrast stimuli, $F(1,5)=29.58$ and $0.51, p=$
.003 and .506 , respectively. The only significant interaction was between SOA and contrast, indicating that the increase in priming with SOA was steeper for high-contrast than for low-contrast stimuli, $F(2,10)=7.91, p=.011$.

Visual awareness. Figure 2B (SOAs of 33-100 ms) shows that prime identification performance depended in a complex way on all experimental variables. An ANOVA of the arcsine-transformed rates of correct identifications showed that prime identification strongly depended on stimulus contrast, with better performance for higher color contrast, $F(1,5)=46.83, p=.001$. Performance was also better for weak than for strong masks, $F(1,5)=8.09, p=$ .036. Identification rates increased with SOA, $F(2,10)=10.23$, $p=.006$, and there was an interaction of SOA $\times$ Contrast, $F(2$, $10)=6.25, p=.017$, reflecting the steeper increase with SOA for high color contrast. The three-way interaction did not reach significance, $F(2,10)=3.93, p=.091$.

Pointing and priming trajectories. We analyzed trajectories in mask ID by projecting the horizontal and vertical coordinates of the fingertip onto a line connecting the red and green mask, such that the starting point was defined as zero, and the correct target direction was defined as positive. Figure 3 shows how the primes affected the trajectories of correct pointing responses. After the primes and masks had occurred, the finger remained at rest for a while. About $200-250 \mathrm{~ms}$ after prime onset, it started to move in the direction specified by the primes. In consistent trials, this was the correct direction; the finger traveled continuously in the direction of the correct target until the response was completed. In inconsistent trials, however, the finger made a detour into the quadrant specified by the misleading prime, stopped, and then commenced in the correct direction. This detour was longer the more time had elapsed between primes and masks. Trajectories were analyzed by four ANOVAs with factors of time bin (in 25-ms

\section{Prime identification vs. priming effects}
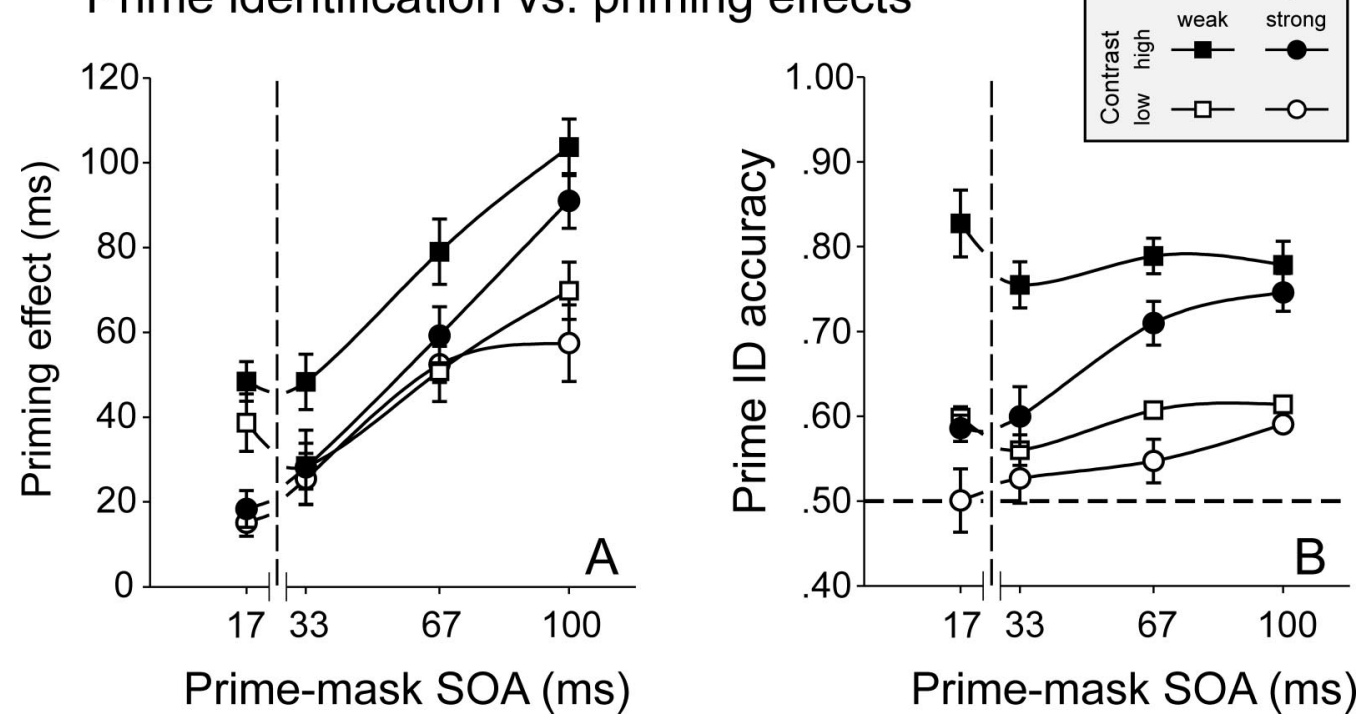

Figure 2. A: Priming effects in arrival times. B: Prime identification performance. Note that in both panels, data from the 17-ms stimulus onset asynchrony (SOA) come from Experiment 2, and data from the remaining SOAs come from Experiment 1. Error bars denote standard errors of the mean with pure intersubject variance removed. 


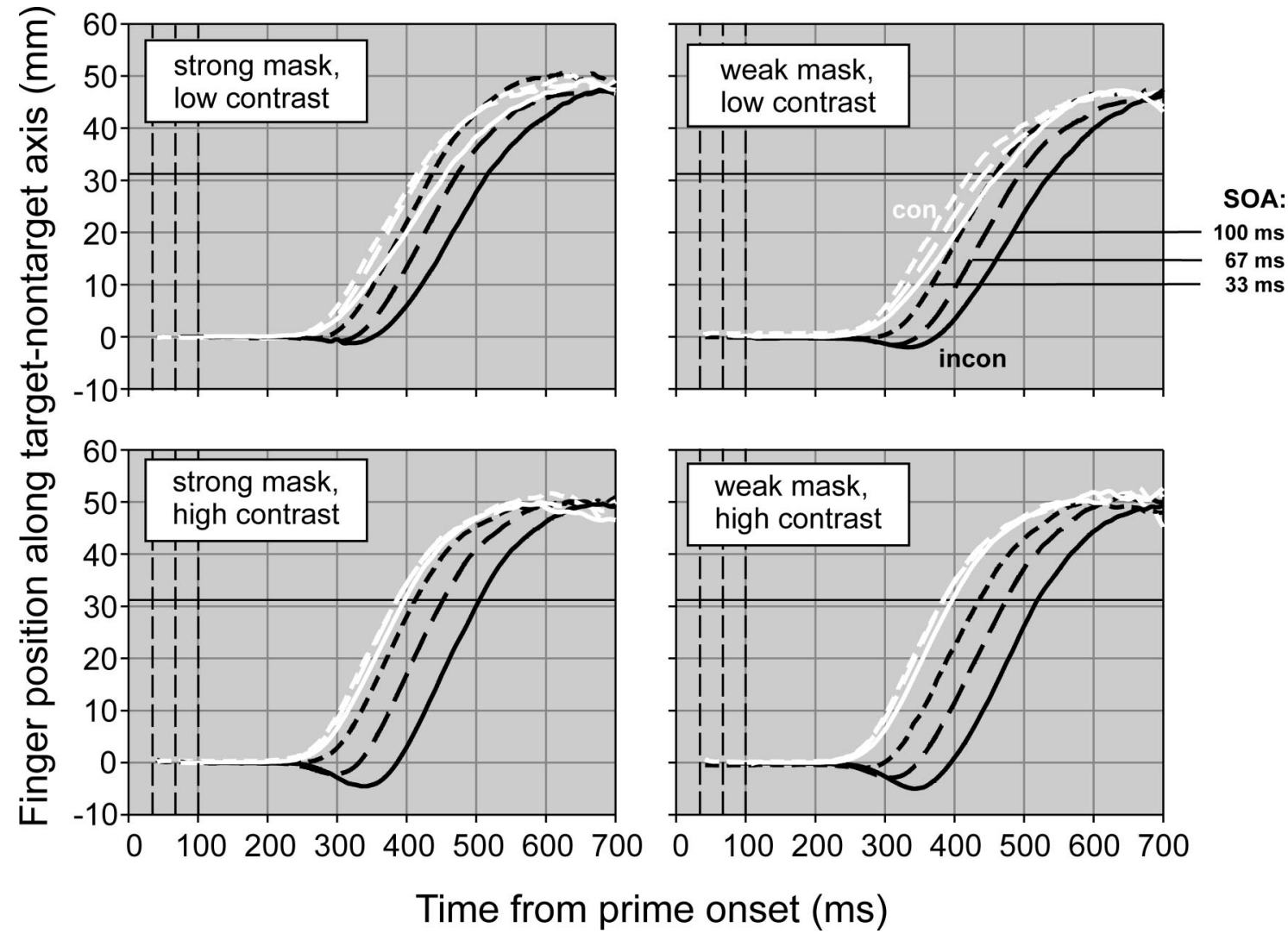

Figure 3. Pointing trajectories of correct responses in Experiment 1. Three-dimensional finger position was projected onto the line connecting the correct and incorrect target. Positive values indicate responses in the direction of the correct target; negative values indicate detours into the quadrant opposite the correct one. The time axis is locked to prime onset; dashed vertical lines show mask onset times for the different stimulus onset asynchronies (SOAs); the dashed horizontal line marks the arrival criterion. con = consistent; incon = inconsistent.

steps), consistency, and SOA, one analysis for each of the panels in Figure 3. As expected, all interactions and main effects (including the intercept terms) were significant (all $p s \leq .003$ ).

We established a spatial measure of the priming effect (see Figure 4) by subtracting Figure 3's trajectories in consistent trials from those in inconsistent trials (negative values indicating how far the finger position in inconsistent trials lags behind the finger position in consistent trials at corresponding time samples). As expected, these priming trajectories were strictly time-locked to the occurrence of the primes rather than the masks. Unexpectedly, however, the early time courses of priming were virtually identical for all SOA and masking conditions at a given contrast level, and only branched off from the common time course at different points in time. To see the significance of this striking invariance, which was obvious in each participant, consider a general model of priming in which the pointing movement at the onset of the priming effect would be controlled by information coming from the mask as well as the prime. Under such a model, the initial slopes of the priming trajectories should be smaller for shorter prime-mask SOAs and for stronger masks, because these factors should increase the influence of the mask signal relative to the prime signal and thus reduce the priming effect. In contrast, the finding that the early time course of the priming effect is invariant indicates that the mask signal has no influence whatsoever at the time when the prime first affects the pointing movement. In other words, early priming effects must be based on signals carrying only prime but no mask information.

Priming trajectories were analyzed with two ANOVAs with factors of time bin (150-500 ms from prime onset, in 25-ms steps), SOA, and mask type, one for each of the two panels in Figure 4. Both analyses confirmed effects of time bin, SOA, and an interaction of both factors (all $p \mathrm{~s} \leq .001$ ). For high-contrast stimuli, there was an additional effect of mask type, $F(1,5)=111.31, p<$ .001 , which did not reach significance for the low-contrast stimuli $(p=.097)$, as well as interactions of the masking factor with SOA, $F(2,10)=6.83, p=.014$, and time bin, $F(14,70)=7.94, p<$ .001 , which were nonsignificant for the low-contrast stimuli (both $p \mathrm{~s}>.251)$.

To evaluate the amount of time the different priming trajectories were running together, we performed interaction contrasts at each 8.33-ms sample point for each pair of priming trajectories, checking whether the pair of curves differed in priming at that point in time. To yield maximum sensitivity, the contrasts were calculated as ANOVAs across single trials rather than participants, control- 


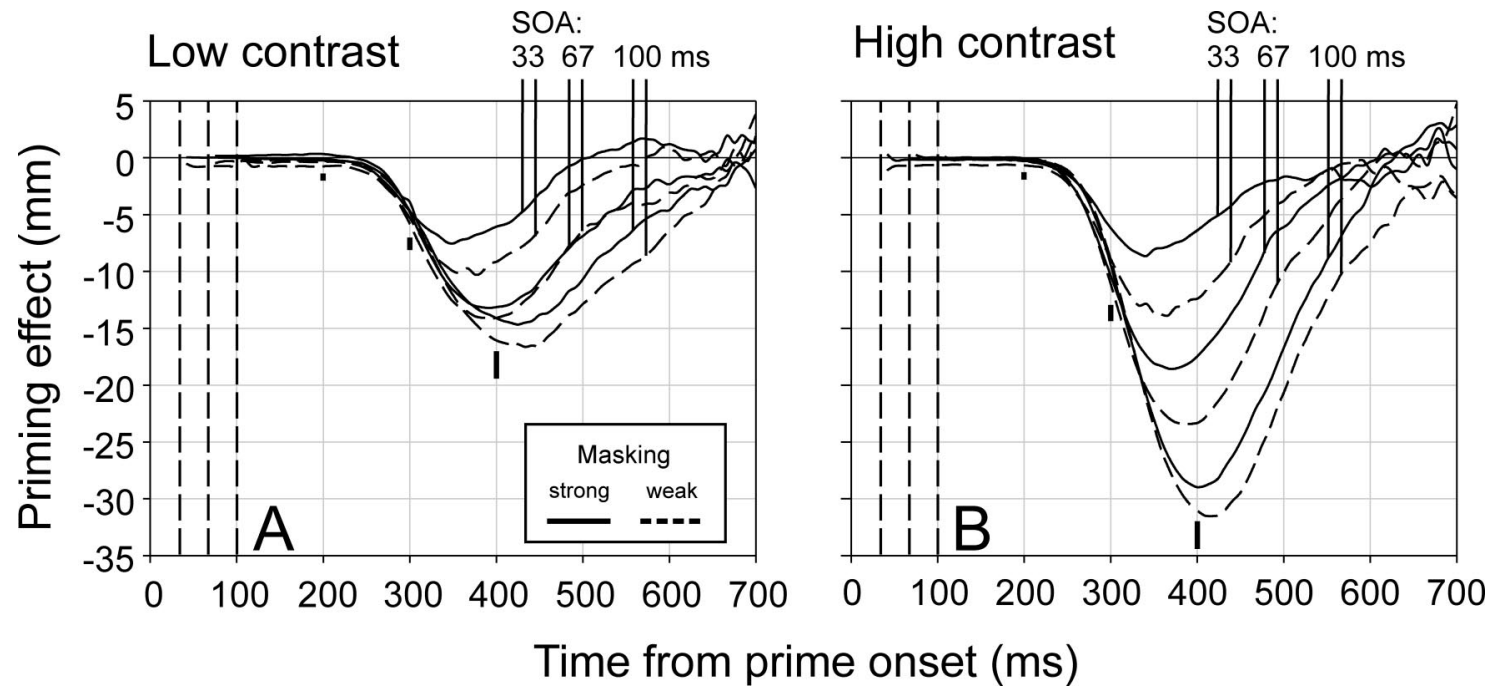

Figure 4. Time course of spatial priming effects (defined as the difference between corresponding consistent and inconsistent trajectories). Negative values indicate how far (in millimeters) the finger in inconsistent trials lags behind the finger in consistent trials at corresponding time samples. Bars below the curves denote one within-subject standard error (pooled across participants) for the 100-ms, weak-mask condition at 200, 300, and $400 \mathrm{~ms} . \mathrm{SOA}=$ stimulus onset asynchrony.

ling for participants by including them as a fixed factor. ${ }^{2}$ Curves were considered to be reliably different as soon as they started to differ at $\alpha \leq .100$ and remained significant at that level until at least $450 \mathrm{~ms}$ after prime onset. Results are shown in Table 1. Priming trajectories separated early for short SOAs and strong masks but late for long SOAs and weak masks. Priming trajectories for the longest SOAs ran together for as long as 150-200 ms following the onset of the priming effect.

Detailed analyses of single trials revealed that pointing movements did not detour into the wrong quadrant in each inconsistent trial (see Figure 5). Instead, detours occurred only in a minority of responses with very fast onsets. These responses always started off in the direction of the primes, and did so in equal proportions for consistent and inconsistent masks, again showing that the earliest overt pointing responses were exclusively driven by the primes. Some responses in inconsistent trials developed into errors; those that did not were corrected online. In the majority of trials, however, priming was manifest in delayed onset times rather than actual detours, indicating that response activation by the primes alone was typically insufficient to trigger an overt response.

\section{Experiment 2}

Experiment 1 shows that an overt detour into the wrong quadrant is the exception rather than the rule, suggesting that covert motor activation processes play a major role in response priming. To look deeper into these early, covert response dynamics, we performed a second experiment that was identical to the first one but used only a single prime-mask SOA of $17 \mathrm{~ms}$. At such a short time interval, the feedforward sweep might lack the temporal resolution to propagate prime and mask signals as consecutive events: Two stimulus signals engaged in a rapid chase would no longer be transmitted as clearly separate, but tend to overlap in time, forming an ambiguous compound signal. Under such condi- tions, inconsistent primes should not lead to detours into the wrong quadrant because the compound signal could not be clearly mapped onto either response. Instead, inconsistent primes should delay the onsets of pointing movements by the time needed for the persisting mask signal to overcome the initially ambiguous compound signal. In addition, even the earliest phase of the priming effect might be influenced by properties of the masks.

Arrival times and visual awareness. Figure 2A (17-ms SOA) shows that substantial priming effects could be obtained despite the short SOA. Priming effects were analyzed with a repeatedmeasures ANOVA with factors of mask type and color contrast There was strong evidence for response priming, as shown by the intercept effect, $F(1,4)=35.74, p=.004$. Priming effects were slightly larger for high-contrast than for low-contrast stimuli, $F(1$, $4)=9.236, p=.038$, and substantially larger for weak than for strong masks, $F(1,4)=32.63, p=.005$, without any interaction. Similar effects were observed in the error rates. An ANOVA of the arcsine-transformed accuracy rates in prime ID confirmed that identification performance depended on both mask type and color contrast (see Figure 2B, 17-ms SOA). Prime identification was better for weak than for strong masks, $F(1,4)=16.75, p=.015$,

\footnotetext{
${ }^{2}$ Including participants as a fixed rather than a random factor means that we can generalize from the sample of trials to the population of trials only for those participants included in the experiment, instead of generalizing from a sample of participants to a population of participants (Cronbach, Gleser, Nanda, \& Rajaratnam, 1972). This restriction in generalizability comes along with a huge gain in statistical power for detecting even small effects, because the denominator degrees of freedom in the analysis are now determined by the number of trial repetitions rather than by the number of participants. That power is needed here because we have to argue in favor of a null hypothesis stating that priming functions are not statistically different.
} 
Table 1

First Sample Points (Milliseconds After Prime Onset) at Which Priming Functions Become and Remain Significantly Different at $p<.10$

\begin{tabular}{lccccc}
\hline $\begin{array}{c}\text { Stimuli and } \\
\text { sample points }\end{array}$ & Strong-33 & Weak-33 & Strong-67 & Weak-67 & Strong-100 \\
\hline $\begin{array}{c}\text { Low contrast } \\
\text { Weak-33 }\end{array}$ & 333.33 & & & & \\
Strong-67 & 316.67 & 358.33 & & & \\
Weak-67 & 300.00 & 333.33 & never & & \\
Strong-100 & 325.00 & 358.33 & never & 458.33 & \\
Weak-100 & 316.67 & 341.67 & 383.33 & 391.67 & never \\
High contrast & & & & & \\
Weak-33 & 283.33 & 308.33 & & & \\
Strong-67 & 275.00 & 291.67 & 308.33 & & \\
Weak-67 & 275.00 & 308.33 & 325.00 & 350.00 & \\
Strong-100 & 291.67 & 308.33 & 325.00 & 341.67 & 400.00 \\
Weak-100 & 291.67 & & & \\
\hline
\end{tabular}

Note. Sampling raster is $8.33 \mathrm{~ms}$. Strong-33 = strong mask, 33-ms stimulus onset asynchrony (SOA); Weak-33 = weak mask, 33-ms SOA; etc. Ideally, values should be homogenous within columns and increase monotonically from left to right.

and also better for high-contrast than for low-contrast stimuli, $F(1$, $4)=8.58, p=.043$. In contrast to the priming data, there was also an interaction of both variables, $F(1,4)=20.08, p=.011$, indicating that the effect of mask type was much stronger when color contrast was high.

Pointing and priming trajectories. Figure 6 shows that inconsistent primes did not lead to notable detours into the wrong quadrant but only delayed the onsets of the pointing movements (see Figures 6A and 6B). Trajectories were analyzed with four ANOVAs with factors of time bin (in 25-ms steps) and consistency, one analysis for each combination of mask type and color contrast. As expected, all interactions and main effects (including the intercept term) were significant (all $p \mathrm{~s} \leq .011$ ). Priming time courses (see Figure 6C) were analyzed with two ANOVAs as in Experiment 1, one for each level of color contrast, with factors of time bin (150-450 ms from prime onset, in 25-ms steps) and mask type. Despite the short SOA, there were surprisingly large priming effects at each level of color contrast, as indicated by significant intercept terms (both $p \mathrm{~s} \leq .03$ ). Each analysis confirmed strong effects of time bin and strong interactions of time bin and mask type (all $p$ s $<.001$ ), reflecting the stronger dynamics of the priming effects for weak masks. However, the main effect of mask type indicating that priming was stronger for weak than for strong masks was reliable only for high-contrast stimuli, $F(1,4)=$ 254.31, $p<.001$, but not for low-contrast stimuli, $F(1,4)=7.37$, $p=.53$.

In each contrast condition, priming functions for weak and strong masks separated early. For high-contrast stimuli, the time sample where priming in strong-mask and weak-mask conditions first differed at $\alpha<.100$ (see Experiment 1) occurred as early as $225 \mathrm{~ms}$ after prime onset; for the low-contrast stimuli, it was at 275 ms. The separation was discernible right from the onset of the priming effect; there was little evidence that the priming trajectories followed the same early time course. Therefore, even the earliest phases of the priming effect seem to be influenced by mask type provided that the prime-mask SOA is sufficiently short.

\section{Discussion}

Consistent with earlier results, color primes produced priming of speeded pointing responses to subsequent color masks (Schmidt, 2002). Response priming effects increased monotonically with prime-mask SOA. Inconsistent primes delayed arrival times by activating a pointing response in the direction of the misleading prime (i.e., the quadrant opposite the correct one). The onset of the pointing response was locked to prime onset, and the responses beginning earliest invariably started in prime direction, producing a detour into the wrong quadrant. Most of these movements reversed mid-flight after a time depending on the prime-mask SOA; only some of them led to full-fledged errors.

The most surprising finding here is that the initial time course of priming effects in trajectories is virtually identical for both types of masks and for all SOAs studied. This invariance provides strong evidence for an early visuomotor signal that exclusively carries prime information but is uncontaminated by subsequent mask information. Further analyses show that this invariance also applies to conditions in which there is no discernible detour into the wrong quadrant, so that the inconsistent primes simply delay the onset of a movement in correct direction (e.g., the shortest SOAs in the low-contrast conditions in Experiment 1). Even here, the delay depends exclusively on properties of the primes and is independent of any properties of the masks. The invariance of early priming effects breaks down only when the prime-mask SOA becomes extremely short, as in Experiment 2.

The kinematics of the priming effect are further modulated by two variables. First, higher color contrast leads to earlier onsets of pointing responses, larger peak priming effects, and a higher probability of detours into the wrong quadrant. This is consistent with the notion that higher color contrast leads to more vigorous feedforward activation, which in turn leads to a faster build-up of both prime- and mask-related motor activation. Second, priming is enhanced by weak compared with strong masks, possibly because the thin pseudomasks trigger a weaker feedforward sweep by 
Direction:
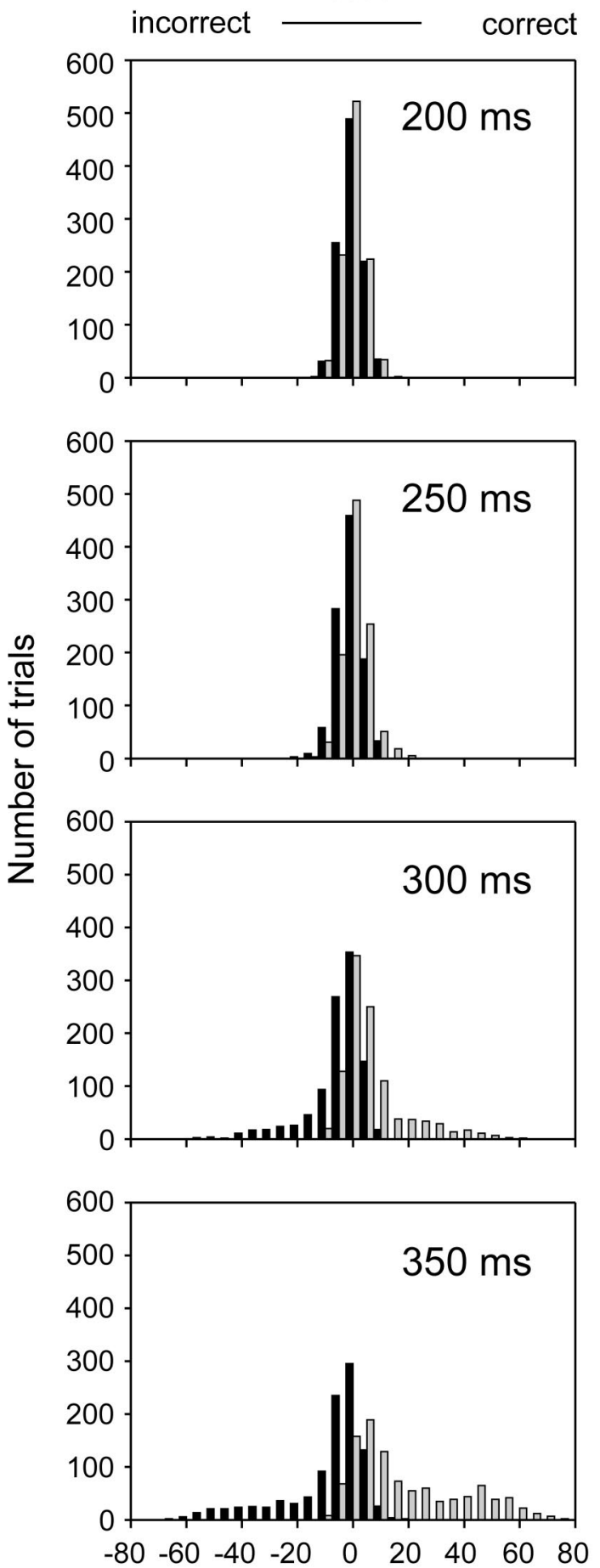

Direction:

incorrect correct
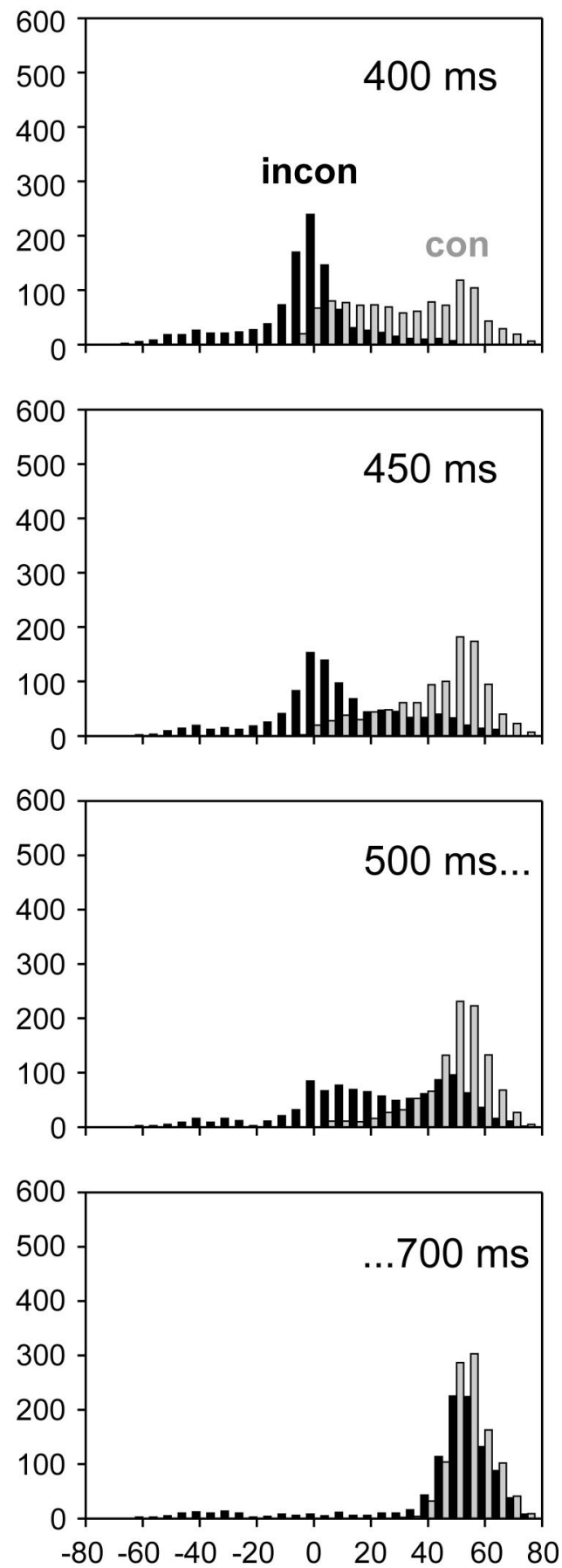

Finger position along target-nontarget axis $(\mathrm{mm})$

Figure 5. Spatial distributions of finger positions at different times after prime onset for consistent (shaded bars) and inconsistent (filled bars) trials. Trajectories are normalized so that the direction of the correct target is plotted rightward. Data are from the condition producing the strongest priming effects (100-ms stimulus onset asynchrony, high color contrast, pooled over weak and strong masks) and include correct as well as incorrect responses. Distributions are based on the projections of finger positions onto the line connecting the correct and incorrect target. 

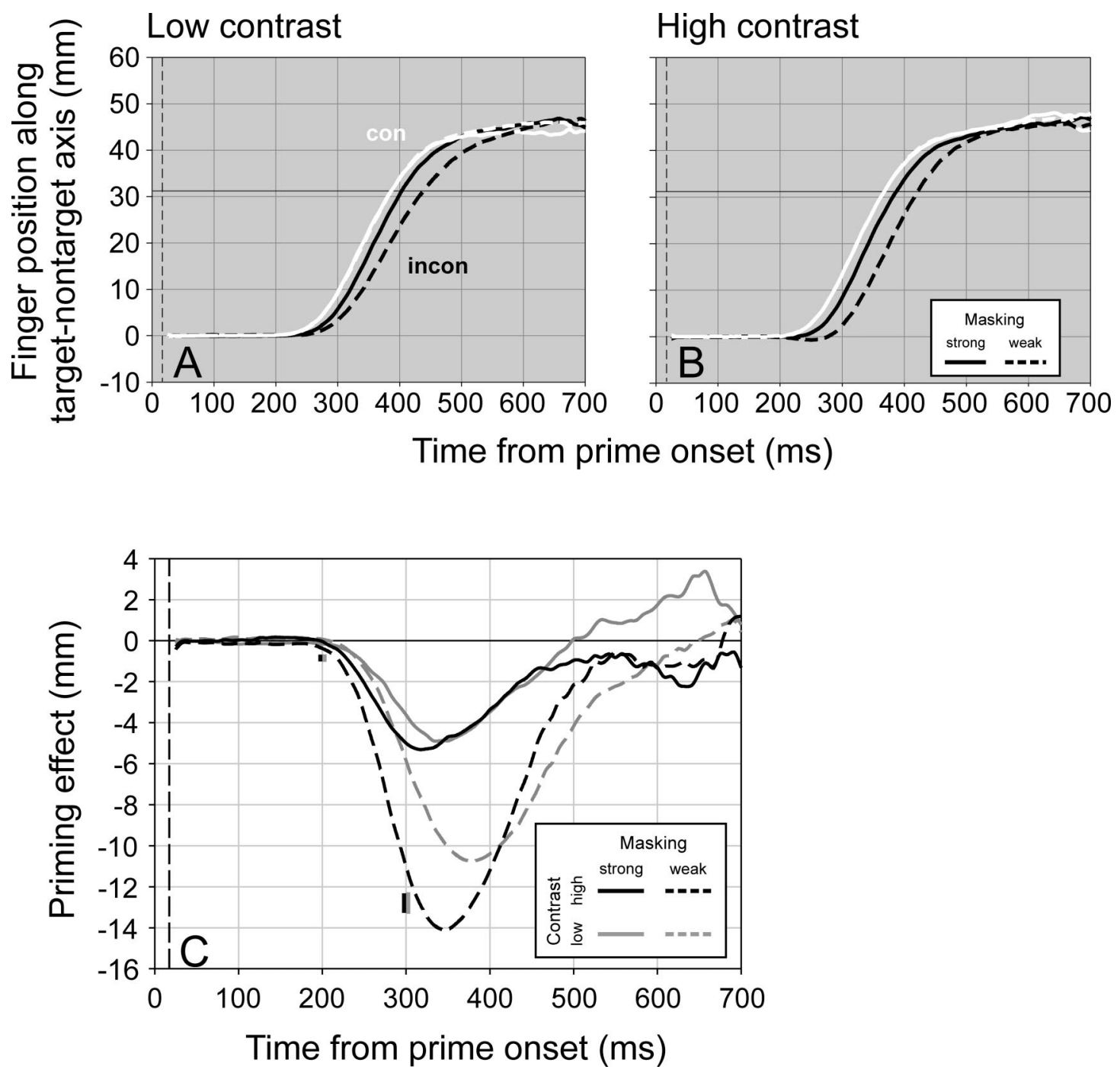

Figure 6. A and B: Pointing trajectories in Experiment 2. Three-dimensional finger position was projected onto the line connecting the correct and incorrect target. Positive values indicate responses in the direction of the correct target; negative values indicate detours into the quadrant opposite the correct one. The time axis is locked to prime onset; dashed vertical lines show mask onset times for the different stimulus onset asynchronies (SOAs); the dashed horizontal line marks the arrival criterion. The two consistent trajectories (white) are indiscernible because they are almost identical. C: Time course of spatial priming effects in Experiment 2. Bars below the curves denote one within-subject standard error (pooled across participants) for the weak masking curves (color-coded) at 200 and $300 \mathrm{~ms}$. con = consistent; incon = inconsistent.

covering a smaller spatial area and thus having lower overall energy.

Visual awareness for the primes as measured in the prime ID task is correlated to later phases of the priming effect, such that longer SOAs, weaker masks, and higher color contrast all lead to better prime identification as well as stronger priming effects. Hence, the patterns of arrival times and prime identification rates tend to be influenced by the same variables in the same direction, albeit to different degrees and under low-visibility conditions. This pattern should not be taken to indicate that it is prime visibility as such that determines the size of the priming effect: Response priming in keypress reaction times has been shown to increase with prime-mask SOA independent of whether primes are visible or invisible (Klotz \& Neumann, 1999; Schmidt, 2002; Vorberg et al., 2003) or whether their visibility increases or decreases with SOA (Mattler, 2003; Vorberg et al., 2003), clearly showing that visual awareness of the primes is not a necessary condition for priming to occur. Schmidt and Vorberg (2006) discuss the various types of dissociations that can take place between measures of priming and awareness along with their underlying assumptions. For our purposes here, it is more important that the initial priming trajectories are invariant despite the notable differences in prime identification performance, indicating that at least the earliest phases of the priming effect are independent of visual awareness of the primes. 
Our conclusions are in line with recent data from rapid image classification tasks. Human observers can classify images surprisingly rapidly, for example, when deciding which of two pictures contains an animal, using manual response times (Thorpe, Fize, \& Marlot, 1996) or saccades (Kirchner \& Thorpe, 2006). VanRullen and Koch (2003) studied this task using pictures that were patternmasked at different SOAs. Participants were presented with single images in a go/no-go task, having to respond with a single keypress whenever the picture contained an animal. Strikingly, the distribution of the earliest response times was identical for masked and unmasked stimuli, indicating that only slower responses were affected by the mask. Bacon-Macé, Macé, Fabre-Thorpe, and Thorpe (2005) reported that this result holds not only for response time distributions but also for occipital EEG responses, which are triggered by the image and have identical early time courses. Even though these data show that visuomotor processing is fast enough to escape visual masking (Fehrer \& Raab, 1962), they do not suffice to demonstrate that this rapid processing is feedforward. To make a strong case for feedforward processing, it must be shown that sequential response control to sequential stimuli obeys the rapid chase criteria outlined in this article.

Our data are in opposition to claims that color processing, compared with form processing, is isolated from immediate response control (Cressman, Franks, Enns, \& Chua, 2006; Goodale \& Westwood, 2004; Milner \& Goodale, 1995; Pisella et al., 2000). Support for that claim comes from studies in which ongoing speeded pointing movements have to be interrupted when the target undergoes a sudden change in either color or position (Pisella et al., 2000), or have to be corrected in flight when a red pointing target switches position with a green distractor at pointing onset (Cressman et al., 2006, using a paradigm adapted from Brenner \& Smeets, 2004). These studies found online response modification to be slower and more difficult with color than with position changes, leading the authors to conclude that color signals cannot be used in the automatic guidance of pointing movements.

The effects of color and position changes in the Pisella et al. (2000) and Cressman et al. (2006) studies are difficult to compare because these conditions differ markedly in several features (e.g., luminance transients, attentional capture, and apparent motion). The color conditions in these studies differ from our paradigm in using (approximately) isoluminant red and green targets exchanging colors during the pointing movement, whereas in our studies both primes and targets had a distinct luminance onset that is probably important for providing accurate timing to the motor system. In direct contradiction to Cressman et al.'s conclusions, Brenner and Smeets (2004) have shown that color signals can redirect a pointing movement online with a latency as short as 100 $\mathrm{ms}$, at least under favorable conditions in which the perturbation occurs when the finger is already moving, and the change in movement direction is not large. ${ }^{3}$ Single-cell and brain imaging studies also reveal that cells in some visuomotor areas (e.g., lateral intraparietal cortex) can be color-selective provided that color is a relevant feature for the behavioral task at hand (Claeys et al., 2004; Toth \& Assad, 2002). Such findings run counter to Goodale and Westwood's (2004) assumption that color processing and online response control reside in anatomically or functionally separate brain systems.

However, the findings by Brenner and Smeets (2004) also pose some challenge to our account of response priming. In our exper- iments, observable responses to the prime developed about $220 \mathrm{~ms}$ after stimulus onset, whereas Brenner and Smeets showed that it takes only about $100 \mathrm{~ms}$ for an ongoing pointing movement to adjust to a stimulus perturbation. How does the system allow for rapid chases despite the additional delay? In Brenner and Smeets' task, the finger was already moving when the perturbation occurred, whereas in our experiments the finger had to accelerate from a resting position, which is commonly assumed to require passing an additional integration-to-threshold phase (e.g., Hanes \& Schall, 1996; Schall, Hanes, \& Taylor, 2000). Ignoring the differences in stimulation (and the additional mechanical inertia that had to be overcome in our task), the 120-ms discrepancy seems a plausible estimate of the time to first reach the motor threshold. Our data already indicate that much of the conflicting response activation reflected in response priming tasks occurs during the integration time before the overt response is elicited (see the model by Vorberg et al., 2003). It is likely that the integration threshold was lowered once the response was under way: Otherwise, if a surprise perturbation signal such as Brenner and Smeets' would have to pass the same threshold as the signal that first triggered the response, the latency of online motor adjustments would be severely limited by the time required to re-reach the threshold. Regardless, if an integration phase is indeed involved here, our findings suggest that prime and mask signals independently pass this phase without overlapping or interacting, conforming to our rapid chase criteria.

Our account of response priming extends Neumann's (1990; Neumann \& Klotz, 1994) theory of direct parameter specification (DPS). DPS assumes that participants in a speeded reaction-time task use a fixed stimulus-response mapping established at the time of instruction and practice. When a trial begins, this mapping is already active and waiting for the last visual parameter that will uniquely specify the required response, which is then triggered automatically without the need for conscious mediation. We work from the assumption that this account is basically correct, trying to link its psychological level of explanation to the neurobiological concepts of feedforward and feedback processing. Whereas DPS predicts only that response priming might be independent of visual awareness of the prime, our account additionally predicts that priming should conform to the rapid chase criteria. The data reported in this article nicely fit these criteria: The pointing response is initiated by the prime signal, is initially independent of the mask signal, and is taken over mid-flight by the mask signal.

We propose that possible pointing targets throughout the visual field compete for continuous control of the pointing response, with higher stimulus strength (e.g., contrast, saturation, stimulus energy) leading to stronger feedforward signals, which are associated with lower transmission times and faster build-up of activation in executive motor areas. Prime and mask signals traverse the visuomotor system sequentially. Prime-related information is transmitted rapidly down the visuomotor pathways by a signal that carries only prime and no mask information and is thus independent of the onset time and the type of mask. This signal must consist of the

\footnotetext{
${ }^{3}$ The difference in results is surprising, given the similar methodology in these two studies. The major difference seems to be that Cressman et al (2006) used a shorter pointing distance and much faster movements than did Brenner and Smeets (2004).
} 
first few spikes in the wavefront of neuronal feedforward activity (Rousselet, Fabre-Thorpe, \& Thorpe, 2002; Thorpe, Fize, \& Marlot, 1996; VanRullen \& Thorpe, 2002), because any larger temporal overlap of prime and mask signals would destroy the early invariance of the priming effect. Because the prime-triggered signal has a head start over the mask-triggered one, it is more likely to control the initial phases of movement. We assume that visual awareness as well as visual masking are due to recurrent feedback degrading the prime signal before it reaches awareness (DiLollo et al., 2000; Lamme, Zipser, \& Spekreijse, 2002), but after it has been propagated downstream by feedforward activity. Therefore, low-level stimulus features like contrast or energy will tend to have similar effects on priming and awareness, and visual backward masking will exclusively affect visual awareness.

Overt detours into the wrong quadrant occur when an inconsistent prime is able to trigger a movement in the wrong direction that is either reversed online by the mask signal or develops into a full-fledged error response. This occurs only for those responses that happen to have a very early onset. In the majority of trials, however, mask-triggered response activation kicks in before prime-triggered activation leads to actual movements, and priming effects are largely confined to the threshold-integration phase before the onset of the overt response. When the SOA between prime and mask stimuli is shorter than about $30 \mathrm{~ms}$, prime and mask signals start to overlap in time and form a compound stimulus that is too ambiguous to trigger a detour, so that the movement can start only after the sustained mask-triggered activation has overcome the transient compound signal. When prime and mask signals overlap or mix, the early invariance of the priming trajectories breaks down, and even the earliest phases of observable movements are influenced by properties of the masks.

Lamme and Roelfsema's (2000) feedforward sweep hypothesis seems to provide the best explanation of how behavioral tasks can conform to our definition of a rapid chase. Data from primed pointing movements (and probably a large array of similar tasks) thus provide independent behavioral evidence for an early phase of visuomotor processing that is at least primarily if not entirely feedforward. To the degree that recurrent processing may turn out as a necessary condition for visual awareness, feedforward tasks such as response priming may be useful in studying the properties of visual processing that occurs preconsciously, that is, before feedback loops start to determine what we consciously perceive.

\section{References}

Ansorge, U., \& Neumann, O. (2005). Intentions determine the effect of invisible metacontrast-masked primes: Evidence for top-down contingencies in a peripheral cueing task. Journal of Experimental Psychology: Human Perception and Performance, 31, 762-777.

Bacon-Macé, N., Macé, M. J. M., Fabre-Thorpe, M., \& Thorpe, S. J. (2005). The time course of visual processing: Backward masking and natural scene categorisation. Vision Research, 45, 1459-1469.

Breitmeyer, B. (1984). Visual masking: An integrative approach. Oxford: Oxford University Press.

Brenner, E., \& Smeets, J. B. J. (2004). Colour vision can contribute to fast corrections of arm movements. Experimental Brain Research, 158, 302-307.

Bullier, J. (2001). Integrated model of visual processing. Brain Research: Brain Research Reviews, 36, 96-107.

Claeys, K. G., Dupont, P., Cornette, L., Sunaert, S., Van Hecke, P. De
Schutter, E., \& Orban, G. A. (2004). Color discrimination involves ventral and dorsal stream visual areas. Cerebral Cortex, 14, 803-822.

Cressman, E. K., Franks, I. M., Enns, J. T., \& Chua, R. (2006). No automatic pilot for visually guided aiming based on colour. Experimental Brain Research, 171, 174-183.

Cronbach, L. J., Gleser, G. C., Nanda, H., \& Rajaratnam, N. (1972). The dependability of behavioral measurements: Theory of generalizability for scores and profiles. New York: Wiley.

Dehaene, S., Naccache, L., Le Clec'H, G., Koechlin, E., Mueller, M., Dehaene-Lambertz, G., et al. (1998, October 8). Imaging unconscious semantic priming. Nature, 395, 597-600.

DiLollo, V., Enns, J. T., \& Rensink, R. A. (2000). Competition for consciousness among visual events: The psychophysics of reentrant visual processes. Journal of Experimental Psychology: General, 129, 481-507.

Eimer, M., \& Schlaghecken, F. (2003). Response facilitation and inhibition in subliminal priming. Biological Psychology, 64, 7-26.

Fehrer, E., \& Raab, E. (1962). Reaction time to stimuli masked by metacontrast. Journal of Experimental Psychology, 63, 143-147.

Francis, G. (1997). Cortical dynamics of lateral inhibition: Metacontrast masking. Psychological Review, 104, 572-594.

Girard, P., Hupé, J. M., \& Bullier, J. (2001). Feedforward and feedback connections between areas V1 and V2 of the monkey have similar rapid conduction velocities. Journal of Neurophysiology, 10, 1563-1573.

Goodale, M. A., \& Westwood, D. A. (2004). An evolving view of duplex vision: Separate but interacting cortical pathways for perception and action. Current Opinion in Neurobiology, 14, 203-211.

Hanes, D. P., \& Schall, J. D. (1996, October 18). Neural control of voluntary movement initiation. Science, 274, 427-430.

Kirchner, H., \& Thorpe, S. J. (2006). Ultra-rapid object detection with saccadic eye movements: Visual processing speed revisited. Vision Research, 46, 1762-1776.

Klotz, W., \& Neumann, O. (1999). Motor activation without conscious discrimination in metacontrast masking. Journal of Experimental Psychology: Human Perception and Performance, 25, 976-992.

Lamme, V. A. F. (2000). Neural mechanisms of visual awareness: A linking proposition. Brain \& Mind, 1, 385-406.

Lamme, V. A. F., \& Roelfsema, P. R. (2000). The distinct modes of vision offered by feedforward and recurrent processing. Trends in Neurosciences, 23, 571-579.

Lamme, V. A. F., Zipser, K., \& Spekreijse, H. (2002). Masking interrupts figure-ground signals in V1. Journal of Cognitive Neuroscience, 14, $1044-1053$.

Leuthold, H., \& Kopp, B. (1998). Mechanisms of priming by masked stimuli: Inferences from event-related brain potentials. Psychological Science, 9, 263-269.

Macknik, S. L., \& Haglund, M. M. (1999). Optical images of visible and invisible percepts in the primary visual cortex of primates. Proceedings of the National Academy of Sciences USA, 96, 15208-15210.

Macknik, S. L., \& Livingstone, M. S. (1998). Neuronal correlates of visibility and invisibility in the primate visual system. Nature Neuroscience, $1,144-149$.

Mattler, U. (2003). Priming of mental operations by masked stimuli. Perception \& Psychophysics, 65, 167-187.

Merigan, W. H., \& Maunsell, J. H. R. (1993). How parallel are the primate visual pathways? Annual Review of Neuroscience, 16, 369-402.

Milner, A. D., \& Goodale, M. A. (1995). The visual brain in action. Oxford, England: Oxford University Press.

Neumann, O. (1990). Direct parameter specification and the concept of perception. Psychological Research, 52, 207-215.

Neumann, O., \& Klotz, W. (1994). Motor responses to nonreportable, masked stimuli: Where is the limit of direct parameter specification? In C. Umiltà \& M. Moscovitch (Eds.), Attention and Performance XV (pp. 124-150). Cambridge, MA: MIT Press. 
Pascual-Leone, A., \& Walsh, V. (2001, April 20). Fast backprojections from the motion to the primary visual area necessary for visual awareness. Science, 292, 510-512.

Pisella, L., Gréa, H., Tilikete, C., Vighetto, A. Desmurget, M., Rode, G., et al. (2000). An 'automatic pilot' for the hand in human posterior parietal cortex: Toward reinterpreting optic ataxia. Nature Neuroscience, 3, 729-736.

Ro, T., Breitmeyer, B., Burton, P., Singhal, N. S., \& Lane, D. (2003). Feedback contributions to visual awareness in human occipital cortex. Current Biology, 11, 1038-1041.

Rousselet, G. A., Fabre-Thorpe, M., \& Thorpe, S. J. (2002). Parallel processing in high-level categorization of natural images. Nature Neuroscience, 5, 629-630.

Schall, J. D., Hanes, D. P., \& Taylor, T. L. (2000). Neural control of behavior: Countermanding eye movements. Psychological Research, 63, 299-307.

Schmidt, T. (2002). The finger in flight: Real-time motor control by visually masked color stimuli. Psychological Science, 13, 112-118.

Schmidt, T., \& Vorberg, D. (2006). Criteria for unconscious cognition: Three types of dissociation. Perception \& Psychophysics, 68, 489-504.

Thorpe, S. J., Fize, D., \& Marlot, C. (1996, June 6). Speed of processing in the human visual system. Nature, 381, 520-522.
Toth, L. J., \& Assad, J. A. (2002, January 10). Dynamic coding of behaviourally relevant stimuli in parietal cortex. Nature, 415, 165-168.

VanRullen, R., \& Koch, C. (2003). Visual selective behavior can be triggered by a feed-forward process. Journal of Cognitive Neuroscience, $15,209-217$.

VanRullen, R., \& Thorpe, S. J. (2002). Surfing a spike wave down the ventral stream. Vision Research, 42, 2593-2615.

Vath, N., \& Schmidt, T. (2006). Tracing sequential waves of visuomotor feedforward activation in lateralized readiness potentials. Manuscript submitted for publication.

Verleger, R., Jaskowski, P., Aydemir, A., van der Lubbe, R. H. J., \& Groen, M. (2004). Qualitative differences between conscious and nonconscious processing? On inverse priming induced by masked arrows. Journal of Experimental Psychology: General, 133, 494-515.

Vorberg, D., Mattler, U., Heinecke, A., Schmidt, T., \& Schwarzbach, J. (2003). Different time courses for visual perception and action priming. Proceedings of the National Academy of Sciences USA, 100, 62756280.

Received December 8, 2005

Revision received May 30, 2006

Accepted June 28, 2006

\title{
ORDER FORM
}

\author{
Start my 2007 subscription to Behavioral \\ Neuroscience! ISSN: 0735-7044
}

\section{- $\$ 139.00$, APA Member/Affiliate \\ $\$ 259.00$, Indrvidual NoNMEMBER \\ $\$ 762.00$, INSTITUTION \\ In DC add $5.75 \%$ / In MD add $5 \%$ sales tax}

Total Amount Enclosed

$\$$

Subscription orders must be prepaid. (Subscriptions are on a calendar year basis only.) Allow 4-6 weeks for delivery of the first issue. Call for international subscription rates.

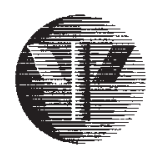

AMERICAN PSYCHOLOGICAL ASSOCIATION

\section{SEND THIS ORDER FORM TO:}

American Psychological Association

Subscriptions

750 First Street, NE

Washington, DC 20002-4242

Or call 800-374-2721, fax 202-336-5568.

TDD/TTY 202-336-6123.

For subscription information, e-mail: subscriptions@apa.org
Check enclosed (make payable to APA)

Charge my: $\bigcirc$ VISA $\bigcirc$ MasterCard $\bigcirc$ American Express

Cardholder Name

Card No. Exp. Date

Signature (Required for Charge)

BILLING ADDRESS:

Street

City State Zip

Daytime Phone

E-mail

MAIL TO:

Name

Address

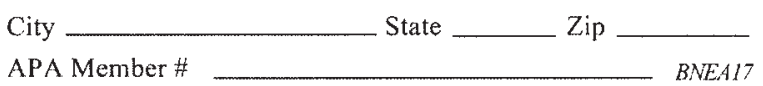

